\title{
There is no generalization of known formulas for mutually unbiased bases
}

\author{
Claude Archer \\ Université Libre de Bruxelles, \\ C.P.165/11 -Physique et Mathématique \\ Faculté des Sciences Appliquées \\ avenue F.D. Roosevelt 50, \\ 1050 Bruxelles, Belgium \\ carcher@ulb.ac.be
}

\begin{abstract}
In a quantum system having a finite number $N$ of orthogonal states, two orthonormal bases $\left\{a_{i}\right\}$ and $\left\{b_{j}\right\}$ are called mutually unbiased if all inner products $\left\langle a_{i} \mid b_{j}\right\rangle$ have the same modulus $1 / \sqrt{N}$. This concept appears in several quantum information problems. The number of pairwise mutually unbiased bases is at most $N+1$ and various constructions of $N+1$ such bases have been found when $N$ is a power of a prime number. We study families of formulas that generalize these constructions to arbitrary dimensions using finite rings. We then prove that there exists a set of $N+1$ mutually unbiased bases described by such formulas, if and only if $N$ is a power of a prime number.
\end{abstract}




\section{Introduction}

\subsection{Definitions and previous results}

In the $\mathrm{N}$-dimensional Hilbert space $\mathbb{C}^{N}$, two orthonormal bases $\left\{a_{i}\right\}_{1 \leq i \leq N}$ and $\left\{b_{j}\right\}_{1 \leq j \leq N}$ are called mutually unbiased if all inner products $\left\langle a_{i} \mid b_{j}\right\rangle$ have the same modulus $\left|\left\langle a_{i} \mid b_{j}\right\rangle\right|=1 / \sqrt{N}$. A set of mutually unbiased bases is a set of orthonormal bases which are pairwise mutually unbiased. In various physical situations (see subsection 1.2), the problem is to find the maximal number of mutually unbiased bases. The following result is due to W.K. Wootters and B.D. Fields but it has been obtained independently by A. R. Calderbank, P. J. Cameron, W. M. Kantor and J. J. Seidel.

Theorem 1.1 ([1], 4])

- In dimension $N \geq 2$, the number of mutually unbiased bases is at most $N+1$.

- If $N$ is a power of a prime number then there exist $N+1$ mutually unbiased bases.

In dimension $N$, a set of mutually unbiased bases is called complete if it contains $N+1$ bases. If $N$ is not a prime power, it is not known whether such a complete set exists, even for $N=6$. Originally, constructions of $N+1$ mutually unbiased bases in dimension $N$, were based on the arithmetic of a field, where addition and multiplication are invertible ([1]). There exists a field with $N$ elements if and only if $N$ is a power of a prime number. Nevertheless, new constructions have been recently obtained (see [8]) using the arithmetic of rings, where multiplication is not invertible. Since there exist rings of $N$ elements for any $N$, is it possible to use finite rings to construct $N+1$ mutually unbiased bases for arbitrary dimensions ? We will address this issue here. First we will generalize known constructions from [11] and [8] to any finite ring. Then we will prove that for dimensions $N$ that are not prime powers, there does not exist a complete set of mutually unbiased bases described by this generalization.

\subsection{Applications to Quantum Information}

Mutually unbiased bases ( $M U B$ for short) have recently been considered with an increasing interest because of the central role they play in specific quantum information tasks. MUB are related, among others, to state estimation and to protocols of quantum cryptography. 
State estimation. Mutually unbiased bases play an important role in state estimation of (relatively) large ensembles of identical prepared quantum systems. MUB allow us to minimize the number of measurements needed to estimate a quantum state. The density matrix of an $N$-dimensional quantum state is determined by $N^{2}-1$ real parameters. Hence, at least $N+1$ measurements are needed to re-construct such a density matrix. One can show that $N+1$ measurements are sufficient if these measurements are MUB ([7]). The reason is that if two measurement bases $B_{1}$ and $B_{2}$ are mutually unbiased, then the information revealed by the outcomes of these measurements are independent. Other optimality properties of MUB with respect to state estimation are described in [11].

Quantum cryptography. The protocol BB84 of Bennett and Brassard (3]) for quantum key distribution, used with the one-time pad encryption, is the first cryptographic protocol whose security does not depend on the assumption that an eavesdropper has a limited computational power. Its security is guaranteed by Heisenberg's uncertainty principle.

MUB are the basic algebraic structure underlying $d$-dimensional analogues of the BB84 protocol ([5]). It is precisely the use of such bases which allows these protocols to make the intervention of a potential eavesdropper detectable. Alice and Bob agree on a set of $t$ orthonormal bases and Alice sends to Bob a state $a_{i}$ prepared in a basis $A=\left\{a_{i}\right\}$ taken among the $t$ bases. Bob chooses a basis in one of the $t$ bases to measure this state. If Bob chooses the right basis, he finds the good value. Now suppose an eavesdropper Eve has chosen $E=\left\{b_{j}\right\}$ to measure the state. Eve obtains $b_{j}$ as result with probability $\left|\left\langle a_{i} \mid b_{j}\right\rangle\right|^{2}$. Since for security purpose, one wants that when $E$ is the wrong base, Eve gets no information on the original state, we should require that all probabilities $\left|\left\langle a_{i} \mid b_{j}\right\rangle\right|^{2}$ are equal and hence equal to $1 / N$ (i.e. $A$ and $E$ are mutually unbiased). Moreover, as the probability to choose a wrong basis is $1 / t$, one wants to take the largest possible number of mutually unbiased bases. It is also known that a protocol using a larger number of mutually unbiased bases can tolerate a higher error level in the channel (see [5] ).

Wigner functions Pure or mixed quantum states are usually represented by the density matrix. However, there is an alternative description in terms of the Wigner function. Several authors have proposed to define a Wigner function for discrete systems having $N$ degrees of freedom. It appears that the discrete Wigner function defined in [12] requires the existence of $N+1$ mutually unbiased bases.

Finally, MUB have also been shown to be relevant to the mean king's 
problem, see [1] and references therein. An interesting source for recent results and for references is the problem page in Quantum Information at TU Braunschweig, located at http://www.imaph.tu-bs.de/qi/problems.

\section{Formulas for mutually unbiased bases}

A unitary transformation maps a set of MUB to a set of MUB. Hence, it is not restrictive to consider only sets $X$ of MUB containing the standard basis $\left\{e_{k}\right\}_{1 \leq k \leq N}$ since it is always possible to choose a unitary transformation $U$ that maps a given orthonormal basis in $X$ to $\left\{e_{k}\right\}$ so that $U(X)$ is a set of MUB containing $\left\{e_{k}\right\}$. If a basis $\left\{v_{k}\right\}$ is unbiased with respect to the standard basis $\left\{e_{k}\right\}$ (i.e. $\left.\left|\left\langle e_{i} \mid v_{j}\right\rangle\right|=1 / \sqrt{N}\right)$ then $\left|\left(v_{k}\right)_{l}\right|=\left|\left\langle e_{l} \mid v_{k}\right\rangle\right|=1 / \sqrt{N}$. Hence the coordinates of its vectors must be expressed as $\left(v_{k}\right)_{l}=\left(e^{i \Theta(k, l)}\right) / \sqrt{N}$ where $\Theta(k, l)$ belongs to $[0,2 \pi]$.

For $N=p^{n}$ where $p$ is a prime number and $n$ a positive integer, there always exist $N+1$ mutually unbiased bases. We describe here the constructions from [11] and 8] for these dimensions.

\subsection{Odd prime powers dimensions}

Let the superscript $r$ denotes the basis, $k$ the vector in the basis and $l$ the component. The standard basis is $\left(v_{k}^{(0)}\right)_{l}=\delta_{k l}$ for $k, l=0,1, \ldots, N-1$. If $N=p^{n}$ for a prime number $p \neq 2$, the other $N=p^{n}$ such bases given in [1] are,

$$
\left(v_{k}^{(r)}\right)_{l}=\frac{1}{\sqrt{N}} e^{(2 \pi i / p) \operatorname{Tr}\left(r l^{2}+k l\right)} \quad r, k, l \in \mathbb{F}_{p^{n}}
$$

where $\mathbb{F}_{p^{n}}$ is the finite field with $p^{n}$ elements and where $\operatorname{Tr}$ denotes the trace map from $\mathbb{F}_{p^{n}}$ into the prime field $\mathbb{F}_{p}$. For $p \geq 5$ odd, a new formula has been proposed in [8] where the polynomial $r l^{2}+k l$ is replaced by $(l+r)^{3}+k(l+r)$. The trace map is a linear map from $\mathbb{F}_{p^{n}}$, regarded as a vector space, into $\mathbb{F}_{p}$. In the language of group theory, linear maps are group homomorphisms ( i.e. maps that preserve sums) . The trace map induces a homomorphism from the additive group of $\mathbb{F}_{p^{n}}$ into the multiplicative group $\mathbb{C}^{*}$ of complex numbers, defined by $x \rightarrow e^{(2 \pi i / p) \operatorname{Tr}(x)}$. 


\subsection{Even prime powers dimensions}

For $N=2^{n}$, W.K.Wootters and B.D.Fields ([1]) have used an ad hoc construction that may be reformulated in a finite ring $R$ whose $4^{n}$ elements are sequences $\left(x_{1}, \ldots, x_{n}\right)$ with $x_{i} \in \mathbb{Z}_{4}$. A much easier construction has been found recently by A. Klappenecker and M. Roetteler ([8]) using the Galois ring $R=G R(4, n)$. Let $\operatorname{Tr}$ denotes the trace map from $G R(4, n)$ into $\mathbb{Z}_{4}$. Once again $T: x \rightarrow e^{(2 \pi i / 4) \operatorname{Tr}(x)}$ is a group homomorphism from $G R(4, n),+$ into $\mathbb{C}^{*}$. The $2^{n}$ indexes are the elements of $T_{n}$, the Teichmüller set of $G R(4, n)$ and the $2^{n}$ bases described by

$$
\left(v_{k}^{(r)}\right)_{l}=\frac{1}{\sqrt{2^{n}}} e^{(2 \pi i / 4) \operatorname{Tr}((r+2 k) l)} \quad r, k, l \in T_{n} \subset G R(4, n)
$$

together with the standard basis, form a complete set of mutually unbiased bases of $\mathbb{C}^{2^{n}}$ (see [8]).

\subsection{How to generalize these formulas ?}

Formulas (11), (21) as well as others in [8], share many common characteristics. First of all, the indexes $l, k, r$ respectively for components, vectors and bases are taken in a finite ring $R$. Both formulas link the indexes in $R$ to complex coordinates by a function $f:(r, k, l) \rightarrow T(P(r, k, l))$ where $P$ is a polynomial and $T$ is a homomorphism from $R,+$ into $\mathbb{C}^{*}$. We will generalize these characteristics as follows :

1. The functions $f:(r, k, l) \rightarrow T(P(r, k, l))$. We consider a much larger class of functions that we call functions preserving a direct sum decomposition of $R$ (see section 44).

2. The set $S$ of indexes. For formula (11) the set of indexes is the whole $R$ while for formula (2), it is a remarkable subset of $R$. We will see in subsection 2.5 that these subsets may be defined for every ring $R$ as sets closed under multiplication and transversal to a nilpotent ideal of $R$.

3. Distinguish the index $r$. In formula (11) and (2), the non standard bases are indexed by $r$ that takes all possible values of a set of size $N$. This can only be done for dimensions $N$ for which there exist $N+1$ mutually unbiased bases. However there is, up to now, no result showing that this is true if $N$ is not a prime power. Therefore, we propose to give up formulas that are a uniform with respect to $r$ and to consider that each basis $r$ may be described by a different formula. This means that 
for each $r$, we choose a different function $f_{r}:(k, l) \rightarrow f_{r}(k, l)$ into $\mathbb{C}^{*}$ such that the vectors in basis $r$ are described by

$$
\left(v_{k}^{(r)}\right)_{l}=f_{r}(k, l) \quad k, l \in S
$$

where each $f_{r}$ preserve a given decomposition $R_{1} \oplus R_{2}$ and $S \subset$ is as described in previous paragraph. For the case of polynomials $P$ and homomorphisms $T$, it amounts to choose for each $r$, different polynomials $P_{r}$ and homomorphism $T_{r}$.

\subsection{Properties of rings}

In this section we recall various properties of rings that are needed for this paper.

Direct sums of rings. Let $R_{+, \text {. }}$ be a ring where addition is commutative but where multiplication is not necessarily commutative. If the additive group $R_{+}$is the direct sum $R_{1} \oplus R_{2}$ of two subgroups then every element $r$ of $R$ can be written in a unique way as $r=r_{1}+r_{2}$ where $r_{i} \in R_{i}$ and $R_{1} \cap R_{2}$ is reduced to the zero element. An element $r \in R$ may be represented as a couple $\left(r_{1}, r_{2}\right)$ and addition in $R$ corresponds to componentwise addition of couples. If moreover, $R_{1}$ and $R_{2}$ are two-sided ideals of $R$ (i.e. $r \cdot R_{i}=R_{i}=R_{i} \cdot r$ for every $r$ in $R$ ), then multiplication in $R$ is also reduced to componentwise multiplication of couples. Indeed, for $r_{i} \in R_{i}$, $r_{1} . r_{2}$ belongs to both $R_{1}$ and $R_{2}$ since these are two-sided ideals and thus $r_{1} \cdot r_{2}=0=R_{1} \cap R_{2}$. For two ring elements $x$ and $y$, we obtain for their product $x \cdot y=\left(x_{1}+x_{2}\right) \cdot\left(y_{1}+y_{2}\right)=x_{1} \cdot y_{1}+x_{1} \cdot y_{2}+x_{2} \cdot y_{1}+x_{2} \cdot y_{2}$ and since $x_{i} . y_{j}=0$ for $i \neq j$, we get $x . y=x_{1} \cdot y_{1}+x_{2} . y_{2}$. Thus $(x . y)_{i}=x_{i} \cdot y_{i}$. Observe also that $a \cdot r_{1}=\left(a_{1}+a_{2}\right) \cdot x_{1}=a_{1} \cdot x_{1}$ for every $a \in R$. We say that the $\operatorname{ring} R$ is the direct sums of its ideals $R_{1}$ and $R_{2}$. These properties also hold when $R$ is the direct sum of more than two ideals.

Polynomial functions in a ring. If a ring is a direct sum, then let us show that polynomial functions in $R$ may be evaluated componentwise. A monomial on the set of variables $\{x, y, \ldots\}$ is a finite product of elements of this set. In a commutative ring $R$, a polynomial $P(x, y, \ldots)+r_{0}$ is defined as a linear combination $P$ of monomials (on $\{x, y, \ldots\}$ ) with coefficients in $R$ and of $r_{0} \in R$. A polynomial $P+r_{0}$ defines a polynomial function $(x, y, \ldots) \rightarrow P(x, y, \ldots)+r_{0}$ that maps n-uples of $R^{n}$ to elements of $R$. In the non commutative case, this definition is not very convenient since generally $x . r . y \neq r . x . y$ so that products of polynomials would not 
in general be polynomials. Hence we prefer to define a non commutative polynomial function as $P:(x, y, \ldots) \rightarrow r_{0}+\sum_{k} m_{k}(x, y, \ldots)$ where $m_{k}(x, y, \ldots)=w_{k}(x, y, \ldots ; a, b, \ldots)$ is a finite product of non commuting variables in $\{x, y, \ldots\}$ and of coefficients from a set $\{a, b, \ldots\} \subset R$.

Assume now that the ring $R$ is a direct sum $R=R_{1} \oplus R_{2}$ and for $r \in R$, let $r=r_{1}+r_{2}$ be the corresponding decomposition. We have shown in previous paragraph that products in $R$ may be performed componentwise. Thus, for each term $w_{k}$ of a polynomial function $w_{k}(x, y, \ldots ; a, b, \ldots)$ is equal to $w_{k}\left(x_{1}, y_{1}, \ldots ; a_{1}, b_{1}, \ldots\right)+w_{k}\left(x_{2}, y_{2}, \ldots ; a_{2}, b_{2}, \ldots\right)$. As $x_{1} \cdot a_{1}=x_{1} . a$ and $a_{1} \cdot x_{1}=a \cdot x_{1}$ for $a, x \in R$ we may conclude that $w_{k}\left(x_{i}, y_{i}, \ldots ; a_{i}, b_{i}, \ldots\right)$ is equal to $w_{k}\left(x_{i}, y_{i}, \ldots ; a, b, \ldots\right)$, for $i=1,2$, so that $m_{k}(x, y, \ldots)=m_{k}\left(x_{1}, y_{1}, \ldots\right)+$ $m_{k}\left(x_{2}, y_{2}, \ldots\right)$. Hence, for a polynomial function $\bar{P}=P+r_{0}$ on $R_{1} \oplus R_{2}$, we have $P(x, y, \ldots)=P\left(x_{1}, y_{1}, \ldots\right)+P\left(x_{2}, y_{2}, \ldots\right)$ and thus for $\lambda=-r_{0}=$ $\bar{P}(0,0, \ldots)$,

$$
\bar{P}(x, y, \ldots)=\lambda+\bar{P}\left(x_{1}, y_{1}, \ldots\right)+\bar{P}\left(x_{2}, y_{2}, \ldots\right) .
$$

The Sylow decomposition of a finite ring. Let $R$ be a finite ring and let $|R|=\prod_{i} p_{i}^{e_{i}}$ be the factorization of its order into powers of distinct prime numbers. The additive group $R,+$ is a finite commutative group. Hence, it is equal to the direct product $\oplus_{i} \operatorname{Syl}\left(p_{i}\right)$ of its Sylow subgroups and thus every element $r$ of $R,+$ can be written in a unique way as $r=\sum r_{i}$ where $r_{i} \in S_{p_{i}}:=S y l\left(p_{i}\right)$. We call the element $r_{i}$, the $p_{i}$-component of $r$ and it is the unique element contained in the intersection $S_{p_{i}} \cap\left\{r+\left(\oplus_{j \neq i} S_{p_{j}}\right)\right\}$ ( see [6], chapter 3). These subgroups may be defined as $S_{p_{i}}:=\left\{x: p_{i}^{e_{i}} x=0\right\}$ where $p_{i}^{e_{i}} x$ is the repeated sum of $p_{i}^{e_{i}}$ terms $x$. The subgroups $S_{p_{i}}$ are two-sided ideals of the ring $R$, i.e. $r . S_{p_{i}}=S_{p_{i}} \cdot r=S_{p_{i}}$ for every $r \in R$. This is due to the right and left distributive property of a ring since $p_{i}^{e_{i}}(r \cdot x)=\underbrace{r \cdot x+\ldots+r \cdot x}_{p_{i}^{e_{i}} \text { terms }}=$ $r(\underbrace{x+\ldots+x}_{p_{i}^{e_{i}} \text { terms }})=r \cdot\left(p_{i}^{e_{i}} x\right)=r .0=0$ if $x \in S_{p_{i}}$ so that $r . x \in S_{p_{i}}$ and similarly $x . r \in S_{p_{i}}$. Hence every finite ring is the direct sum of its Sylow ideals and a finite ring that is not decomposable as a non trivial direct sum, must be of prime power order. Moreover, if $|R|=d_{1} \cdot d_{2}$ is the product of two coprime numbers $(\geq 2)$ then $R=R_{1} \oplus R_{2}$ where $R_{i}:=\oplus_{p \mid d_{i}} \operatorname{Syl}(p)$.

Ring with unity. From now on, we mainly consider rings $R$ containing a multiplicative unity 1 such that $x .1=x=1 . x$ for every $x \in R$. If $R=\oplus_{i \in I} R_{i}$ has a unity 1 and $R_{i} \neq\{0\}$ then $1_{i}$ is the unity of $R_{i}$. An element $x$ has a left inverse $x_{L}$ (resp. right inverse $x_{R}$ ) if $x_{L} \cdot x=1$ (resp $x \cdot x_{R}=1$ ). An element that has both a left and a right inverse is called a unit. If $x$ is a unit, then 
the inverse $x^{-1}$ is unique since $x_{L}=x_{L} \cdot\left(x \cdot x_{R}\right)=\left(x_{L} \cdot x\right) \cdot x_{R}=x_{R}$. The set $U(R)$ of all units of $R$ is a multiplicative group and by the componentwise multiplication $U\left(R_{1} \oplus R_{2}\right)=U\left(R_{1}\right) \oplus U\left(R_{2}\right)$. A field is a ring where every non zero element is a unit.

Nilpotency. An element $n$ of a ring $R$ is called nilpotent if $n^{t}=0$ for some positive integer $t$. The set $\operatorname{Nil}(R)$ of all nilpotent element of $R$ is called the Nilpotent radical of $R$. Once again, by the componentwise multiplication $\operatorname{Nil}\left(R_{1} \oplus R_{2}\right)=\operatorname{Nil}\left(R_{1}\right) \oplus \operatorname{Nil}\left(R_{2}\right)$. We say that an ideal $N$ is nilpotent provided that every $n \in N$ is nilpotent. For every $r \in R$, if we have $(r . n)^{t}=0$ then $(n . r)^{t+1}=n .(r . n)^{t} \cdot r=0$ and thus every nilpotent ideal is two-sided. In every ring with unity, a nilpotent element cannot be a unit but if $n$ is nilpotent $\left(n^{t}=0\right)$ then $1+n$ is a unit. To show this, consider $u_{t}(n)=1+n+\ldots+n^{t-1}$; then since $1=1-n^{t}=(1-n) u_{t}(n)=u_{t}(n)(1-n)$, the element $u_{t}(-n)$ is the inverse of $1+n=1-(-n)$.

Let us show that in a commutative $\operatorname{ring}, \operatorname{Nil}(R)$ is an ideal. If $x^{n}=0$ then $(r \cdot x)^{n}=r^{n} \cdot x^{n}=0$ and if moreover $y^{m}=0$ then $(x+y)^{n+m}=0$ since $(x+y)^{n+m}$ is a sum of terms $x^{n+m-k} \cdot y^{k}$ which are zero for $k \leq m$ and for $k \geq m$. In a non commutative $\operatorname{ring} R, N i l(R)$ is not necessarily an ideal. For instance, a sum of nilpotent matrices may be invertible (and thus non nilpotent) as shown by

$$
\left(\begin{array}{ll}
0 & 1 \\
1 & 0
\end{array}\right)=\left(\begin{array}{ll}
0 & 0 \\
1 & 0
\end{array}\right)+\left(\begin{array}{ll}
0 & 1 \\
0 & 0
\end{array}\right)
$$

Thus for the ring $M_{2}(R)$ of $2 \times 2$ matrices over a ring $R$ with unity, the nilpotent radical is not an ideal. However the subring of upper triangular matrices is also non commutative but it contains the nilpotent ideal $\left\{\left(\begin{array}{ll}0 & r \\ 0 & 0\end{array}\right): r \in R\right\}$.

\subsection{Generalizing Teichmüller sets}

For every ring $R$ we would like to define a subset $S_{R} \subset R$ for the indexes $k, l$ of vectors and components, in such a way that for a Galois ring $R=G R(4, n)$, the set $S_{R}$ is the Teichmüller set $T_{n}$ as in formula (2), while for a finite field $R=\mathbb{F}_{p^{n}}$ we have $S_{R}=R$ as in formula (11).

The set $T_{n} \subset G R(4, n)$ has remarkable properties that are used over and over to compute easily in $G R(4, n)$ (see [9]).

1. The ideal $N:=\left\{2 t_{1}: t_{1} \in T_{n}\right\}$ is the nilpotent radical of $G R(4, n)\left(N^{2}=0\right)$.

2. Every $r \in G R(4, n)$ can be written in a unique way as $r=t_{o}+2 t_{1}$ for some $t_{0}, t_{1}$ in $T_{n}$. Thus $T_{n}$ contains exactly one representative of each coset $\{r+N\}_{r \in R}$ of $N$ in $R$; it is a transversal to the ideal $N$. 
3. $T_{n}$ is closed under multiplication. Therefore a product of elements written as $t+n$ for $t \in T_{n}$ and $n \in N$ is still written in this way since $\left(t_{1}+n_{1}\right)\left(t_{2}+n_{2}\right)=\underbrace{t_{1} t_{2}}_{\in T_{n}}+(\underbrace{t_{1} n_{2}+n_{1} t_{2}+n_{1} n_{2}}_{\in N})$.

This may be generalized to every ring $R$ as follows. We require that the set of indexes $S_{R}$ is closed under multiplication and that it is a transversal to a nilpotent ideal $N$. Trivially, if $R$ is a field (as in formula (1)) or even a division ring, then $N=\{0\}$ is the only nilpotent ideal, $S_{R}=R$ is the only transversal to $N$ and it is closed under multiplication.

A commutative local ring is a ring that has a unique maximal ideal $M$ and the Galois ring $R=G R(4, n)$ is local. In a finite commutative local ring $R$, the unique maximal ideal is $\operatorname{Nil}(R)$ and the units of $R$ are exactly the non nilpotent elements (see [2]). Hence in a local ring every ideal $(\neq R)$ is nilpotent. Every finite commutative ring with unity is a direct sum of local rings (see [2], Proposition 8.7).

\subsection{Functions preserving a direct sum decomposition}

Let $R=R_{1} \oplus R_{2}$ be a direct sum decomposition of a ring $R$ and let $r=r_{1}+r_{2}$ be the corresponding decomposition for $r \in R$. Let $G,_{\star}$ be a commutative group with an operation $\star$ ( either " + " or ". " in this paper). For a finite set of variables $\{x, y, \ldots\}$ belonging to $R$, we say that a function $f:(x, y, \ldots) \rightarrow$ $f(x, y, \ldots) \in G,_{\star}$ preserves the decomposition $R_{1} \oplus R_{2}$ if for a constant $\lambda \in R$

$$
f(x, y, \ldots)=\lambda \star f\left(x_{1}, y_{1}, \ldots\right) \star f\left(x_{2}, y_{2}, \ldots\right) \quad \text { for every } \quad x, y, \ldots \in R .
$$

Observe that $\lambda=(f(0,0, \ldots))^{-1}$ because $\left(x_{1}\right)_{2}=\left(y_{1}\right)_{2}=\ldots=0$ implies $f\left(x_{1}, y_{1}, \ldots\right)=f\left(x_{1}+0, y_{1}+0, \ldots\right)=\lambda \star f\left(x_{1}, y_{1}, \ldots\right) \star f(0,0, \ldots)$. If $f_{i}$ is the restriction of $f$ to $R_{i}$ then $f(x, y, \ldots)=\lambda \star f_{1}\left(x_{1}, y_{1}, \ldots\right) \star f_{2}\left(x_{2}, y_{2}, \ldots\right)$. Conversely, for arbitrary functions $f_{i}$ from $R_{i}$ into $G$ and $\lambda \in G$, this last equation defines a function that preserves $R_{1} \oplus R_{2}$. It may happen that $f$ preserves $R_{1} \oplus R_{2}$ but does not preserve another decomposition of $R$.

We have seen in subsection 2.4 that polynomial functions $P$ on a ring $R$ preserves every direct sum decomposition of $R$ ( and in this case $\lambda=$ $-P(0,0, \ldots))$. Thus, since group homomorphisms preserve sums, if $T$ is a group homomorphism from $R_{+}$into a commutative group $G_{\star}$ and if $P(x, y, \ldots)$ is a polynomial function on $R$, then $(x, y, \ldots) \rightarrow T(P(x, y, \ldots))$ preserves 
every direct sum decomposition of $R$. Hence, these functions generalize formula (11) and (2) for mutually unbiased bases since those rely on expressions of type $\frac{1}{\sqrt{N}} T(P(k, l))$ for $k, l \in R$ where $G_{\star}$ is the multiplicative group of unitary complex number. This is also true for the other formulas proposed in $[8]$.

More sophisticated such functions may be constructed by products. If $f \cdot g$ is a product of functions into a commutative group $G$. that both preserve a direct sum decomposition $R=R_{1} \oplus R_{2}$ then it is easy to show that $f \cdot g$ also preserves $R_{1} \oplus R_{2}$. We have $(f \cdot g)(x, y, \ldots)=\lambda_{f} \cdot \prod_{i=1,2} f\left(x_{i}, y_{i}, \ldots\right)$. $\lambda_{g} \prod_{i=1,2} g\left(x_{i}, y_{i}, \ldots\right)$ and since the elements of $G$ commute, we may rearrange the factors as $(f \cdot g)(x, y, \ldots)=\lambda_{f} \cdot \lambda_{g} \prod_{i=1,2}(f \cdot g)\left(x_{i}, y_{i}, \ldots\right)$.

\section{Such sets of MUB cannot be complete}

In this section we prove that even with all these generalizations, it is not possible to construct complete sets of $N+1$ mutually unbiased bases for $N \neq p^{n}$.

\subsection{Preliminary results}

Proposition 3.1 In a ring $R$ with 1 , let $S \subset R$ be a set closed under multiplication that is a transversal to a nilpotent ideal $N$ of $R$.

1. If $R=R_{1} \oplus R_{2}$ is a sum of rings with 1 then

$$
S=\left(S \cap R_{1}\right) \oplus\left(S \cap R_{2}\right)
$$

and each $S \cap R_{i}$ contains at least two elements $(i=1,2)$. Moreover in each $R_{i}, S \cap R_{i}$ is closed under multiplication and is a transversal to $N \cap R_{i}$.

2. If $|S|$ is a product $d_{1}$. $d_{2}$ of two coprime numbers $\geq 2$ and if $R$ is finite, then $R$ is a sum $R_{1} \oplus R_{2}$ of rings with 1 such that $\left|S \cap R_{1}\right|=d_{1}$ and $\left|S \cap R_{2}\right|=d_{2}$.

Proof. 1) $R=R_{1} \oplus R_{2}$ has unity $\left(1_{1}, 1_{2}\right)$. First, we show that every ideal $I$ of $R$ is equal to $I_{1} \oplus I_{2}$ where $I_{j}$ is an ideal of $R_{j}$. Since $I$ is an ideal of $R$, the sets $I_{1}:=I .\left(1_{1}, 0\right)$ and $I_{2}:=I .\left(0,1_{2}\right)$ belong to $I$, are ideals of $R$ and thus in particular $I_{j}$ is an ideal of $R_{j}$. But since every $i=\left(i_{1}, i_{2}\right) \in I$ is equal to $i .\left(1_{1}, 0\right)+i .\left(0,1_{2}\right)$, we have $I=I_{1} \oplus I_{2}$. By componentwise multiplication, an element $\left(n_{1}, n_{2}\right)$ is nilpotent if and only if each $n_{j}$ is nilpotent in $R_{j}$ 
and the ideal $N$ is the sum $N_{1} \oplus N_{2}$ of two nilpotent ideals. Since $S$ is a transversal to $N$ in $R$, it contains a unique element of each coset of $N$. Let $x$ be the unique element $S \cap\left\{\left(1_{1}, 0\right)+N\right\}$ then $x=\left(1_{1}+n_{1}, n_{2}\right)$ for some $\left(n_{1}, n_{2}\right) \in N_{1} \oplus N_{2}$. Since $N$ is a nilpotent ideal,it is two-sided and we may consider the quotient ring $R / N$ where multiplication of cosets is defined as $(x+N)(y+N)=x \cdot y+N$. In $R / N,\left(\left(1_{1}, 0\right)+N\right)^{2}=\left(1_{1}, 0\right)+N$ so that $x^{2} \in\left(1_{1}, 0\right)+N$ and also $x^{2} \in S$ because $S$ is closed under multiplication. Therefore we have $x^{2}=S \cap\left\{\left(1_{1}, 0\right)+N\right\}=x$ and we have

$$
\left(1_{1}+n_{1}\right)^{2}=\left(1_{1}+n_{1}\right) \quad(1), \quad n_{2}^{2}=n_{2} \quad(2) .
$$

By nilpotency $n_{2}^{t}=0$ for some positive integer $t$ and by (2), $n_{2}^{t}=n_{2}$ whence $n_{2}=0$. By nilpotency of $n_{1},\left(1+n_{1}\right)$ has an inverse $\left(1_{1}+n_{1}\right)^{-1}$ in $R_{1}$. Multiplying both sides of (1) by $\left(1_{1}+n_{1}\right)^{-1}$ gives $\left(1_{1}+n_{1}\right)=1$ whence $n_{1}=0$. Finally $x=\left(1_{1}, 0\right) \in S$ and the symmetric argument for $S \cap\left\{\left(0,1_{2}\right)+N\right\}$ shows that $\left(0,1_{2}\right)$ also belongs to $S$. Thus $(0,0)=\left(1_{1}, 0\right) \cdot\left(0,1_{2}\right) \in S$, and $S \cap R_{1}$ (resp. $S \cap R_{2}$ ) contains at least the two elements $(0,0)$ and $\left(1_{1}, 0\right)$ (resp. $(0,0)$ and $\left.\left(0,1_{2}\right)\right)$.

As $\left\{\left(1_{1}, 0\right),\left(0,1_{2}\right)\right\} \in S$, for every $\left(s_{1}, s_{2}\right) \in S,\left(s_{1}, 0\right)=s .\left(1_{1}, 0\right) \in S \cap R_{1}$ and $\left(0, s_{2}\right)=s .\left(0,1_{2}\right) \in S \cap R_{2}$. Conversely, it remains to show that for every $\left(s_{1}, 0\right) \in S \cap R_{1}$ and $\left(0, s_{2}\right) \in S \cap R_{2}$ we also have $\left(s_{1}, s_{2}\right) \in S$. Since $S$ is a transversal to $N$, it contains a unique element $y=\left(s_{1}+n_{1}, s_{2}+n_{2}\right) \in$ $S \cap\left\{\left(s_{1}, s_{2}\right)+N\right\}$ and $y \cdot\left(1_{1}, 0\right)=\left(s_{1}+n_{1}, 0\right)$ in $S$. As $\left(s_{1}+n_{1}, 0\right)$ and $\left(s_{1}, 0\right)$ are in $S$ and belong the same coset of $N$, these must be equal and $n_{1}=0$. Similarly $n_{2}=0$ so that $y=\left(s_{1}, s_{2}\right) \in S$ whence $S=\left(S \cap R_{1}\right) \oplus\left(S \cap R_{2}\right)$. Finally, let us show that $\left(S \cap R_{i}\right)$ is a transversal to $N_{i}=N \cap R_{i}$ in $R_{i}$. Every coset $\left(r_{1}, 0\right)+N_{1}$ is embedded in $\left(r_{1}, 0\right)+N$ which contains a unique element $s=\left(r_{1}+n_{1}, n_{2}\right)$ of $S$. Then $s .\left(1_{1}, 0\right)=\left(r_{1}+n_{1}, 0\right)$ is in $S \cap R_{1}$ and in $\left(r_{1}, 0\right)+N_{1}$ and so, $S \cap R_{1}$ contains at least one representative of each coset. If two elements of $S \cap R_{1}$ belong to the same coset of $N_{1}$ then these belong to the same coset of $N \supset N_{1}$ and thus are equal. The proof is similar for $\left(S \cap R_{2}\right)$.

2 ) Let $\pi(d)$ denote the set of prime divisors of a positive integer $d$. For a finite ring $R$ and a divisor $d$ of $|R|$, let us define $\operatorname{Syl}(\pi(d)):=\oplus_{p \in \pi(d)} \operatorname{Syl}(p)$. Since $N$ is a transversal to the ideal $N$ in $R$ then $d_{1} \cdot d_{2}=|S|=\frac{|R|}{|N|}$ is a divisor of $|R|$. If $\pi_{1}=\pi\left(d_{1}\right)$ and $\pi_{2}=\pi(|R|) \backslash \pi\left(d_{1}\right)$, we know from subsection 2.4 that $R=\operatorname{Syl}\left(\pi_{1}\right) \oplus \operatorname{Syl}\left(\pi_{2}\right)$. For $i=1,2$, since $d_{i} \geq 2$, the subsets $\left.\pi_{(} d_{i}\right)$ are non empty so that $\pi_{1}=\pi\left(d_{1}\right)$ and $\pi_{2} \supset \pi\left(d_{2}\right)$ are non empty ; whence the rings $\operatorname{Syl}\left(\pi_{i}\right)$ are not zero rings and since $R$ has unity $1, \operatorname{Syl}\left(\pi_{i}\right)$ has unity $1_{i}$. Thus part (1) of the present proposition applies, $S=\left(S \cap S y l\left(\pi_{1}\right)\right) \oplus\left(S \cap \operatorname{Syl}\left(\pi_{2}\right)\right)$ 
and

$$
d_{1} \cdot d_{2}=|S|=\left|S \cap S y l\left(\pi_{1}\right)\right| \cdot\left|S \cap S y l\left(\pi_{2}\right)\right| .
$$

As $S \cap \operatorname{Syl}\left(\pi_{i}\right)$ is a transversal to the ideal $N \cap \operatorname{Syl}\left(\pi_{i}\right)$ then $\left|S \cap S y l\left(\pi_{i}\right)\right|$ divides $\operatorname{Syl}\left(\pi_{i}\right)$ and so is coprime to $d_{j}(j \neq i)$. Thus, by equality (5), $\left|S \cap S y l\left(\pi_{i}\right)\right|$ must divides $d_{i}$ and symmetrically $d_{i}$ divides $\left|S \cap S y l\left(\pi_{i}\right)\right|$ so that $d_{i}=\left|S \cap S y l\left(\pi_{i}\right)\right|$.

In what follows, $\langle\mid\rangle$ denotes the classical hermitian product $\langle a, b\rangle=$ $\sum_{i}\left(a_{i}\right)^{*} b_{i}$. The tensor product $v \otimes w$ is defined by $(v \otimes w)_{(i, j)}=a_{i} \cdot b_{j}$ and thus $\left\langle v_{1} \otimes w_{1} \mid v_{2} \otimes w_{2}\right\rangle=\left\langle v_{1} \mid v_{2}\right\rangle .\left\langle w_{1} \mid w_{2}\right\rangle$.

Proposition 3.2 Let $N=N_{1} \cdot N_{2}$ be a product of positive integers and let $\left\{v_{i, j}^{(1)}\right\} \ldots\left\{v_{i, j}^{(r)}\right\}$ be $r$ mutually unbiased bases of $\mathbb{C}^{N} \cong \mathbb{C}^{N_{1}} \otimes \mathbb{C}^{N_{2}}($ for $(i, j) \in$ $\left.\bar{N}:=\left\{1 \ldots N_{1}\right\} \times\left\{1 \ldots N_{2}\right\}\right)$. Assume that for each $1 \leq t \leq r$ there are $N_{1}$ vectors $\left\{a_{i}^{(t)}\right\}$ and $N_{2}$ vectors $\left\{b_{j}^{(t)}\right\}$ such that

$$
v_{i, j}^{(t)}=a_{i}^{(t)} \otimes b_{j}^{(t)}
$$

for every $(i, j) \in \bar{N}$, then for $1 \leq t \leq r,\left\{\frac{a_{i}^{(t)}}{\left\|a_{i}^{(t)}\right\|}\right\}$ and $\left\{\frac{b_{j}^{(t)}}{\left\|b_{j}^{(t)}\right\|}\right\}$ are $r$ mutually unbiased bases respectively in $\mathbb{C}^{N_{1}}$ and $\mathbb{C}^{N_{2}}$.

Proof. First we show that $\left\{a_{i}^{(t)}\right\}$ and $\left\{b_{j}^{(t)}\right\}$ are orthogonal bases. Since othonormality of $\left\{v_{i, j}^{(t)}\right\}$ implies $\left\langle v_{i, j}^{(t)} \mid v_{k, l}^{(t)}\right\rangle=\delta_{\{(i, j),(k, l)\}}=\delta_{i k} \delta_{j l}$, we have $\delta_{i k} \delta_{j l}=\left\langle a_{i}^{(t)} \otimes b_{j}^{(t)} \mid a_{k}^{(t)} \otimes b_{l}^{(t)}\right\rangle=\left\langle a_{i}^{(t)} \mid a_{k}^{(t)}\right\rangle\left\langle b_{j}^{(t)} \mid b_{l}^{(t)}\right\rangle$. For every $j, b_{j}^{(t)} \neq \overline{0}$ (otherwise $v_{i, j}^{(t)}=\overline{0}$ ) so that $\left\langle a_{i}^{(t)} \mid a_{k}^{(t)}\right\rangle\left\langle b_{j}^{(t)} \mid b_{j}^{(t)}\right\rangle=\delta_{i k} \delta_{j j}=\delta_{i k}$ implies that $\left\langle a_{i}^{(t)} \mid a_{k}^{(t)}\right\rangle=0$ for $i \neq k$. Hence $\left\{a_{i}^{(t)}\right\}$ is a set of $N_{1}$ mutually orthogonal vectors, thus an orthogonal basis ( not necessarily orthonormal ) of $\mathbb{C}^{N_{1}}$. Permuting the role of $a$ and $b$ gives the same result for $\left\{b_{j}^{(t)}\right\}$ in $\mathbb{C}^{N_{2}}$.

Furthermore if we fix $1 \leq j \leq N_{2}$, the equalities $1=\left\langle v_{i, j}^{(t)} \mid v_{i, j}^{(t)}\right\rangle=\left\langle a_{i}^{(t)} \mid a_{i}^{(t)}\right\rangle\left\langle b_{j}^{(t)} \mid b_{j}^{(t)}\right\rangle$ may be divided by the constant $\left\langle b_{j}^{(t)} \mid b_{j}^{(t)}\right\rangle$ so that for every $1 \leq i \leq N_{1}$, $L_{a^{(t)}}:=\left\langle a_{i}^{(t)} \mid a_{i}^{(t)}\right\rangle$ is constant with respect to $i$ and equal to $1 /\left\langle b_{j}^{(t)} \mid b_{j}^{(t)}\right\rangle$. Symmetrically, $L_{b^{(t)}}:=\left\langle b_{j}^{(t)} \mid b_{j}^{(t)}\right\rangle$ is also constant for every $1 \leq j \leq N_{2}$ and

$$
L_{a^{(t)}}:=\left\langle a_{i}^{(t)} \mid a_{i}^{(t)}\right\rangle=\frac{1}{\left\langle b_{j}^{(t)} \mid b_{j}^{(t)}\right\rangle}=\frac{1}{L_{b^{(t)}}} \quad \text { for every } \quad(i, j) \in \bar{N} .
$$

Now, it is sufficient to prove the MUB property for each couple of bases among the $\mathrm{r}$, in $\mathbb{C}^{N_{1}}$ and $\mathbb{C}^{N_{1}}$. For instance let us consider $\left\{v_{i, j}^{(1)}\right\}$ and $\left\{v_{k, l}^{(2)}\right\}$. 
We define $A_{i, k}:=\left|\left\langle a_{i}^{(1)} \mid a_{k}^{(2)}\right\rangle\right|$ and $B_{j, l}:=\left|\left\langle b_{j}^{(1)} \mid b_{l}^{(2)}\right\rangle\right|$. The equality

$$
1 / \sqrt{N}=\left|\langle\underbrace{v_{i, j}^{(1)}}_{a_{i}^{(1)} \otimes b_{j}^{(1)}} \mid \underbrace{v_{k, l}^{(2)}}_{a_{k}^{(2)} \otimes b_{l}^{(2)}}\rangle\right|=A_{i, k} B_{j, l} \quad \text { for every }(i, j),(k, l) \in \bar{N}
$$

implies that $A_{i, k}$ and $B_{j, l}$ are non zero. Therefore if $(i, k)$ is fixed and $(j, l)$ varies, $A_{i, k}$ can be simplified and all the $B_{j, l}$ are equal to a common value $K_{B}$. Symmetrically, the $A_{i, k}$ are equal to a common value $K_{A}$.

In basis $\left\{a_{i}^{(1)}\right\}$ we have $a_{k}^{(2)}=\sum_{i} \lambda_{i} a_{i}^{(1)}$ for $\lambda_{i}=\frac{\left\langle a_{i}^{(1)} \mid a_{k}^{(2)}\right\rangle}{\left\langle a_{i}^{(1)} \mid a_{i}^{(1)}\right\rangle}$. Now, equality (6) and (17) prove that $\left|\lambda_{i}\right|=\frac{K_{A}}{L_{a^{(1)}}}$ whence it is constant for every $(i, k)$. Therefore

$$
\begin{gathered}
L_{a^{(2)}}=\left|\left\langle a_{k}^{(2)} \mid a_{k}^{(2)}\right\rangle\right|=\left|\left\langle\sum_{i} \lambda_{i} a_{i}^{(1)} \mid \sum_{i^{\prime}} \lambda_{i^{\prime}} a_{i^{\prime}}^{(1)}\right\rangle\right| \\
=\sum_{i}^{N_{1}}\left|\lambda_{i}\right|^{2}\left|\left\langle a_{i}^{(1)} \mid a_{i}^{(1)}\right\rangle\right|=N_{1}\left|\lambda_{i}\right|^{2} L_{a^{(1)}}=N_{1}\left(\frac{K_{A}}{L_{a}(1)}\right)^{2} L_{a^{(1)}}=\frac{N_{1}\left(K_{A}\right)^{2}}{L_{a^{(1)}}} .
\end{gathered}
$$

$$
\text { that is } L_{a^{(1)}} L_{a^{(2)}}=N_{1}\left(K_{A}\right)^{2}
$$

Finally, we show that $\left\{\frac{a_{i}^{(1)}}{\left\|a_{i}^{(1)}\right\|}\right\}$ and $\left\{\frac{a_{k}^{(2)}}{\left\|a_{k}^{(2)}\right\|}\right\}$ are mutually unbiased bases in $\mathbb{C}^{N_{1}}$. Indeed $\left|\left\langle\frac{a_{i}^{(1)}}{\left\|a_{i}^{(1)}\right\|} \mid \frac{a_{k}^{(2)}}{\left\|a_{k}^{(2)}\right\|}\right\rangle\right|^{2}=\frac{\left|\left\langle a_{i}^{(1)} \mid a_{k}^{(2)}\right\rangle\right|^{2}}{\left\|a_{i}^{(1)}\right\|^{2}\left\|a_{k}^{(2)}\right\|^{2}}=\frac{\left(K_{A}\right)^{2}}{L_{a^{(1)}} L_{a}(2)}=\frac{1}{N_{1}}$ (by (이) ).

The result for $\mathbb{C}^{N_{2}}$ is obtained in the same way, using $b_{l}^{(2)}=\sum_{j} \mu_{j} b_{j}^{(1)}$ for $\mu_{j}=\frac{\left\langle b_{j}^{(1)} \mid b_{l}^{(2)}\right\rangle}{\left\langle b_{j}^{(1)} \mid b_{j}^{(1)}\right\rangle}$, to give $L_{b^{(1)}} L_{b^{(2)}}=N_{2}\left(K_{B}\right)^{2}$.

This proposition can immediately be extended as follows to $\mathbb{C}^{N} \cong \mathbb{C}^{N_{1}} \otimes$ $\ldots \otimes \mathbb{C}^{N_{s}}$ for dimension $N=N_{1} \ldots N_{s}$. Under assumption that each of the $\mathrm{k}$ bases is a tensor product, we may use induction to conclude to the existence of $k$ mutually unbiased bases in each $\mathbb{C}^{N_{i}}$.

\subsection{Main results}

Theorem 3.1 Let $R=R_{1} \oplus R_{2}$ be a decomposition of a ring $R$. For $i=1,2$ let $S_{i}$ be a non empty subset of $R_{i}$ and let $N=\left|S_{1}\right|\left|S_{2}\right|$. For each $1 \leq$ $c \leq m$, let $f_{c}: R_{+} \rightarrow \mathbb{C}^{*}, \cdot$ be a two variables function that preserves the decomposition $R_{1} \oplus R_{2}$ and let us define $N$ vectors $\left\{v_{k}^{(c)}\right\}$ of $\mathbb{C}^{N}$ as

$$
\left(v_{k}^{(c)}\right)_{l}=f_{c}(k, l) \quad k, l \in S_{1} \oplus S_{2} .
$$

Assume that, together with the standard basis, the sets of vectors $\left\{v_{k}^{(c)}\right\}_{1 \leq c \leq m}$ form a set $X$ of $m+1$ mutually unbiased bases. If $\left|S_{i}\right|_{i=1,2} \neq 1$, then 


$$
m \leq \min _{i}\left|S_{i}\right|<N \text { and the set } X \text { is not complete. }
$$

Proof. The function $f_{c}$ preserves the decomposition $R_{1} \oplus R_{2}$ so there is a constant $\lambda_{c}$ such that $f_{c}(k, l)=\lambda_{c} f_{c}\left(k_{1}, l_{1}\right) f_{c}\left(k_{2}, l_{2}\right)$. For each $c$, let us define $\left|S_{1}\right|$ vectors $\left\{a_{k_{1}}^{(c)}\right\}_{k_{1} \in S_{1}}$ of $\mathbb{C}^{\left|S_{1}\right|}$ and $\left|S_{2}\right|$ vectors $\left\{b_{k_{2}}^{(c)}\right\}_{k_{2} \in S_{2}}$ of $\mathbb{C}^{\left|S_{2}\right|}$ as

$$
\left(a_{k_{1}}^{(c)}\right)_{l_{1}}=\lambda_{c} f_{c}\left(k_{1}, l_{1}\right) \quad, \quad\left(b_{k_{2}}^{(c)}\right)_{l_{2}}=f_{c}\left(k_{2}, l_{2}\right) \quad \text { for } \quad k_{i}, l_{i} \in S_{i}(i=1,2) .
$$

Hence $\left(v_{k}^{(c)}\right)_{l}=f_{c}(k, l)=\lambda_{c}\left(a_{k_{1}}^{(c)}\right)_{l_{1}}\left(b_{k_{2}}^{(c)}\right)_{l_{2}}$ and since $l$ takes all value in $S_{1} \oplus$ $S_{2}$, the vector $v_{k}^{(c)}=v_{\left(k_{1}, k_{2}\right)}^{(c)}$ is equal to the tensor product $a_{k_{1}}^{(c)} \otimes b_{k_{2}}^{(c)} \in$ $\mathbb{C}^{\left|S_{1}\right|\left|S_{2}\right|}$. If we denote by $\left\{v_{k}^{(0)}\right\}_{k \in S_{1} \oplus S_{2}},\left\{a_{k_{1}}^{(0)}\right\}_{k_{1} \in S_{1}},\left\{b_{k_{2}}^{(0)}\right\}_{k_{2} \in S_{2}}$ the standard bases respectively in $\mathbb{C}^{N}, \mathbb{C}^{\left|S_{1}\right|}$ and $\mathbb{C}^{\left|S_{2}\right|}$ then also $v_{k}^{(0)}=v_{\left(k_{1}, k_{2}\right)}^{(0)}=a_{k_{1}}^{(0)} \otimes b_{k_{2}}^{(0)}$.

Therefore if the sets $\left\{v_{k}^{(c)}\right\}_{0 \leq c \leq m}$ form a set $X$ of $m+1$ mutually unbiased bases in $\mathbb{C}^{N}=\mathbb{C}^{\left|S_{1}\right|\left|S_{2}\right|}$, then by Proposition 3.2. there exist $m+1$ mutually unbiased bases in both $\mathbb{C}^{\left|S_{1}\right|}$ and $\mathbb{C}^{\left|S_{2}\right|}$. By Theorem 1.1. if each $\left|S_{i}\right|_{i=1,2}$ is at least 2 then $m+1 \leq\left|S_{i}\right|+1$ and $m \leq \min _{i}\left|S_{i}\right|<\left|S_{1}\right|\left|S_{2}\right|=N$ thus $|X|=m+1<N+1$ and $X$ is not complete.

Finally, we obtain our main result : complete sets of MUB described by generalizations of known formulas only exist for prime power dimensions. Moreover, we provide an upper bound for the number of MUB described by such formulas.

Theorem 3.2 Let $R$ be a finite ring with unity. Let $S \subset R$ be a subset of $N$ elements that is closed under multiplication and transversal to a nilpotent ideal. For $1 \leq c \leq N$, let $T_{c}: R_{+} \rightarrow \mathbb{C}^{*}, \cdot$ be a group homomorphism and let $P_{c}: R^{2} \rightarrow R$ be a two variables polynomial function. Let us define $N$ sets of vectors $\left\{v_{k}^{(c)}\right\}_{1 \leq c \leq N}$ in $\mathbb{C}^{N}$ by

$$
\left(v_{k}^{(c)}\right)_{l}=\frac{1}{\sqrt{N}} T_{c}\left(P_{c}(k, l)\right) \quad k, l \in S,
$$

and let the set $X_{\left\{T_{c}\right\}\left\{P_{c}\right\}}$ be the union of the standard basis with $\left\{v_{k}^{(c)}\right\}_{1 \leq c \leq N}$.

1. A set $X_{\left\{T_{c}\right\}\left\{P_{c}\right\}}$ contains at most $1+\min _{i}\left\{p_{i}^{e_{i}}\right\}$ mutually unbiased bases where $N=\prod_{i} p_{i}^{e_{i}}$ is the factorization of $N$ into powers of distinct prime numbers.

2. There exists a complete set $X_{\left\{T_{c}\right\}\left\{P_{c}\right\}}$ of $N+1$ mutually unbiased bases if and only if $N$ is a power of a prime number. 
Proof.

1. We prove that the conditions of Theorem 3.1 applies here.

First we show that every vector $v_{k}^{(c)}$ is unbiased with the standard basis $\left\{e_{k}\right\}$. Every $r \in R$ has finite additive order $n_{r}\left(n_{r} \cdot r=0\right)$. By the homomorphism property $T_{c}(n . r)=T_{c}(\underbrace{r+\ldots+r}_{n \text { terms }})=\left(T_{c}(r)\right)^{n}$ and as $T_{c}(0)=1$ we must have $\left|T_{c}(r)\right|^{n}=1$ whence $\left|T_{c}(r)\right|=1$ for every $r$ in $R$. Thus for every vector $v_{k}^{(c)}$ we obtain $\left|\left\langle e_{l} \mid v_{k}^{(c)}\right\rangle\right|=\left|\left(v_{k}^{(c)}\right)_{l}\right|=$ $\left|T_{c}\left(P_{c}(k, l)\right)\right| / \sqrt{N}=1 / \sqrt{N}$ as announced. Let $m+1$ be the maximal number of mutually unbiased bases contained in $X_{\left\{T_{c}\right\}\left\{P_{c}\right\}}$ and let $Y \subset X_{\left\{T_{c}\right\}\left\{P_{c}\right\}}$ be a set of $m+1$ mutually unbiased bases. Since we have showed that $Y \cup\left\{\left\{e_{k}\right\}\right\}$ is also a set of MUB, the standard basis $\left\{e_{k}\right\}$ must be in $Y$.

As $|S|=N=\prod_{i} p_{i}^{e_{i}}$ we may use Proposition [3.1 (2) to show that there is a ring decomposition $R=\oplus_{i} R_{i}$ such that $S=\oplus_{i} S \cap R_{i}$ and $\left|S \cap R_{i}\right|=p_{i}^{e_{i}}$. Finally, since the functions $(k, l) \rightarrow T_{c}\left(P_{c}(k, l)\right)$ preserve every direct sum decomposition of $R$ (see subsection 4 ), we may apply Theorem 3.1 to $Y$ to show that for every $i$ we must have $m \leq p_{i}^{e_{i}}$. Hence $1+m \leq 1+\min _{i}\left\{p_{i}^{e_{i}}\right\}$.

2. If we have $N+1$ such MUB, then by (1), $\prod_{i} p_{i}^{e_{i}}=N \leq \min _{i}\left\{p_{i}^{e_{i}}\right\}$, which implies that $N=\min _{i}\left\{p_{i}^{e_{i}}\right\}$ and thus $N$ is a prime power. Conversely if $N=p_{i}^{e_{i}}$, we have shown in section 2 that the sets of $N+1$ MUB given by formulas (11) and (2) may be described as sets $X_{\left\{T_{c}\right\}\left\{P_{c}\right\}}$

The bound $1+\min _{i}\left\{p_{i}^{e_{i}}\right\}$ can be easily reached for dimension $N=\prod_{i} p_{i}^{e_{i}}$. It suffices to view $\mathbb{C}^{N}$ as $\otimes_{i} \mathbb{C}^{p_{i}^{e_{i}}}$. As $\left\langle b_{i} \otimes c_{k} \mid b_{j} \otimes c_{l}\right\rangle=\left\langle b_{i} \mid b_{j}\right\rangle \cdot\left\langle c_{k} \mid c_{l}\right\rangle$ we may conclude that a tensor product of two sets with $t$ MUB is a set of $t$ MUB in the product space. Since there exist at least $1+\min _{i}\left\{p_{i}^{e_{i}}\right\}$ MUB in each $\mathbb{C}^{p_{i}^{e_{i}}}$ we may construct by tensor product of these, a set of $1+\min _{i}\left\{p_{i}^{e_{i}}\right\}$ MUB in the product space $\mathbb{C}^{N}$. 


\subsection{Discussion on larger generalizations and conclu- sion}

In order to further generalize formula (3) it could be tempting to allow the index set $S$ to be any subset of a finite ring. Unfortunately, this leads to a situation where any set of vectors could be described by such a formula. To see this, let us recall that functions $f$ that preserve a decomposition of a ring $R$ are arbitrary functions on each component $R_{i}$. If we choose a ring $R$ that has no decomposition (a field for instance) then such a $f$ is arbitrary on $R$. If $S$ is a subset $\left\{s_{1}, \ldots, s_{N}\right\}$ of $N$ elements, then we may associate an arbitrary set of vectors $\left\{v_{k}\right\}_{1 \leq k \leq N}$ in $\mathbb{C}^{N}$ to the couples in $S \times S$ by $\left(s_{k}, s_{l}\right) \rightarrow\left(v_{k}\right)_{l}$.

This may be extended (in many ways) to a two variables function from $R \times R$ into $\mathbb{C}$ that preserves every decomposition of $R$ (since $R$ cannot be decomposed). One cannot expect to reach algebraic conclusions that are valid for all $N \times N$ arrays with arbitrary complex entries $\left(v_{k}\right)_{l}$.

For these reasons, it is difficult to generalize formula (3) much more. It indicates that for dimensions that are not prime powers, algebraic formulas providing complete sets of MUB should have a radically new structure. However, do these complete sets exist for any dimension ? Mathematicians are used to properties that behave differently for some particular dimensions but such an answer is unsatisfactory from a physical point of view. W.K. Wooters has showed that the absence of $N+1$ MUB for a dimension $N$ would be problematic for defining a discrete Wigner function in systems having $N$ degrees of freedom (see [12]). A negative answer to the MUB problem might have other physical consequences and these could be used to guide mathematical investigations.

\section{Acknowledgement}

I gratefully acknowledge Sofyan Iblisdir for introducing me to this problem and for all our fruitful discussions.

\section{References}

[1] Y. Aharonov, B.-G. Englert, The mean king's problem: Spin 1, Z. Naturforsch. 56a, 16-19 (2001).

[2] M. Atiyah, I. McDonald, Introduction to Commutative Algebra, AddisonWesley Pub. Co. (1969). 
[3] Bennett, C.H. and Brassard, G, Quantum Cryptography : Public Key Distribution and Coin Tossing. Proceedings of the IEEE International Conference on Computers, Systems and Signal Processing, IEEE, New York, 175-179 (1984).

[4] A. R. Calderbank, P. J. Cameron, W. M. Kantor, and J. J. Seidel, $\mathbb{Z}_{4}$-Kerdock codes, orthogonal spreads, and extremal Euclidean line-sets, Proc. London Math. Soc. 75, 436-480 (1997).

[5] N. Cerf, M. Bourennane, A. Karlsson, N. Gisin, Security of Quantum Key-Distribution using d-level Systems, Phys. Rev. Lett. 88127902 (2002).

[6] M. Hall Jr., The theory of groups The Macmillan Company (1968).

[7] I. D. Ivanovic, Geometrical description of quantal state determination, $J$. Phys. A 14, 3241-3245 (1981).

[8] A. Klappenecker, M. Roetteler, Constructions of Mutually Unbiased Bases, http://xxx.lanl.gov/abs/quant-ph/0309120 (2003).

[9] B.R. McDonald, Finite rings with Identity, Marcel Dekker Inc. New-York (1974).

[10] Problem page in Quantum Information at TU Braunschweig, http://www.imaph.tu-bs.de/qi/problems.

[11] W.K. Wootters, B.D. Fields, Optimal state-determination by mutually unbiased measurements, Ann. Phys. 191, 363-381 (1989).

[12] W.K. Wootters, Picturing qubits in phase space, IBM Journal of R \& D 48, No. 1 (2004). 